\title{
Aporte de la Melisopalinología al conocimiento de la FLORA MELÍFERA DE UN SECTOR DE LA PROVINCIA FITOGEOGRÁFICA Chaqueña, Argentina
}

\author{
CRISTINA R. SALGADO ${ }^{1,2}$, GELINA PIESZKO² y MARÍA C. TELLERÍA ${ }^{3}$
}

\begin{abstract}
Summary: Melissopalynological contribution to the knowledge of the honey flora in a sector of the Phytogeographic Chaquenian Province, Argentina. The honey flora of a sector of the Occidental y Oriental Chaco phytogeographic province, Argentina, was studied. Availability of plant flowering, considering the botanical origin of pollen grains contained in honey samples was investigated. Plant specimens and honey samples were collected in 20 apiaries during spring and summer of years 2004 to 2007 . The flowering period in the studied region is larger than in other Argentinean regions, extending almost the entire year. Field records revealed the presence of 282 plants species belonging to 65 Angiosperms families being Fabaceae and Asteraceae the most well represented. Two flowering peaks were detected, one between September and December, and the other from February until late March. The first peak of flowering involves a larger number of plant species than the second. Major flowering contribution was produced by native species; woody plants are dominant in spring and herbaceous ones in summer. Although 118 pollen types were identified in the honey samples, the main nectar sources came from a few plants belonging to Asteraceae, Anacardiaceae, Arecaceae, Fabaceae, Rhamnaceae and Zygophyllaceae.
\end{abstract}

Key words: Melliferous flora, blooming, nectar, pollen, honey, Chaco region.

\begin{abstract}
Resumen: La composición y la oferta de floración de la flora melífera de los dos Distritos de la provincia fitogeográfica Chaqueña, fue estudiada a partir del seguimiento de la floración y del análisis de mieles producidas en diferentes momentos de la temporada apícola. La recolección de ejemplares y de muestras de miel fue realizada durante los años 2004 al 2007 en 20 apiarios. En la región estudiada la oferta de floración es más extensa que en otras regiones argentinas, extendiéndose durante la mayor parte del año. A partir de los registros a campo se relevaron 282 especies pertenecientes a 65 familias de Angiospermas, siendo Fabaceae y Asteraceae las más representadas. Se detectaron dos picos de floración, uno entre septiembre y diciembre, y otro desde febrero hasta fines de marzo. El primer pico de floración es más intenso que el segundo. La principal oferta de floración es producida por especies nativas, siendo las leñosas más comunes en primavera y las herbáceas en verano. De los 118 tipos de polen identificados en las mieles, sólo unos pocos representan fuentes nectaríferas importantes, ellos pertenecen a las siguientes familias: Asteraceae, Anacardiaceae, Arecaceae, Fabaceae, Rhamnaceae y Zygophyllaceae.
\end{abstract}

Palabras clave: Flora melífera, floración, néctar, polen, miel, región chaqueña.

\section{INTRODUCCIÓN}

La producción de mieles con valor agregado requiere conocer la composición y la disponibilidad

${ }^{1}$ Facultad de Ciencias Agrarias - UNNE, Sgto. Cabral 2131, 3400. Corrientes, Argentina. E-mail: polenenmiel@ agr.unne.edu.ar

${ }^{2}$ Instituto de Botánica del Nordeste - IBONE - (UNNECONICET), Sgto. Cabral 2131, 3400. Corrientes, Argentina.

${ }^{3}$ Laboratorio de Sistemática y Biología Evolutiva (LASBE). Museo de La Plata, Paseo del Bosque s/n (1900). La Plata, Argentina. de los recursos nectaríferos de una región. En la Argentina, el conocimiento de las plantas proveedoras de néctar derivan mayormente de estudios melisopalinológicos (e.g. Forcone \& Andrada, 2006; Sánchez \& Vignale, 2009) siendo escasos los estudios que incluyen también el seguimiento de la floración a lo largo de la temporada apícola. En este sentido, cabe mencionarse los estudios realizados por Andrada (2003) para el Espinal y Naab \& Tamame (2007) para la provincia del Monte. En la región Chaqueña, en particular, los estudios sobre la flora melífera son escasos al considerar su gran extensión. A partir de observaciones a 
campo efectuadas en el Departamento Capital de la provincia de Corrientes, Maidana (1976) presentó un listado de plantas de interés apícola y el período de floración de las mismas. Basilio \& Noetinger (2002) estudiaron el polen de las mieles de las provincias de Chaco y Formosa, las autoras destacaron la presencia de componentes exóticos y de plantas nativas que caracterizan a las mieles de la región. Cabrera (2006) realizó estudios polínicos en mieles de Formosa y destacó la importancia de la flora nativa como recurso nectarífero. Cabrera et al. (2013) se refirieron a la fenología de las especies con potencial apícola del Distrito Chaqueño Oriental a partir de un relevamiento de la vegetación. En el marco de un proyecto iniciado por el Ministerio de la Producción de la provincia del Chaco, Salgado (2006) publicó una lista de plantas melíferas de dicha provincia con sus nombres científicos y vernáculos, acompañadas con fotografías con el fin de facilitar su identificación por parte de los apicultores.

Dentro de la región fitogeográfica Chaqueña, Chaco es la provincia con mayor desarrollo apícola del nordeste argentino; cuenta con unas 71000 colmenas que producen unas 650 toneladas de miel/año (Fuente: Ministerio de la Producción del Chaco). Actualmente existe un creciente interés por conocer la flora apícola regional a fin de lograr cosechas diferenciadas por su origen botánico. En este marco, el presente trabajo tiene como objetivo identificar las plantas nectaríferas de la región, a través del seguimiento de la floración de las diferentes especies y de su representatividad en las mieles que se van produciendo en diferentes momentos de la temporada apícola. Se pretende así brindar información útil para la producción de mieles contribuyendo a la vez a un aprovechamiento no destructivo de los bosques nativos. Cabe destacar al respecto que actualmente algunos sectores del bosque chaqueño han sido destruidos no sólo mediante el fuego y el desmonte a fin de crear un terreno apto para el cultivo, sino también por el sobrepastoreo del ganado doméstico (Dinerstein et al., 1995).

\section{Materiales y Métodos}

Para llevar a cabo el estudio, fueron seleccionados 20 apiarios ubicados en la provincia del Chaco (Fig.
1). Esta provincia se encuentra comprendida en la Provincia Fitogeográfica Chaqueña (Cabrera, 1976); en ella están representados los distritos oriental y occidental, ambos poseen diferente régimen de lluvias. El Distrito oriental posee clima húmedo con lluvias que alcanzan los $1200 \mathrm{~mm}$ anuales, en cambio el occidental posee clima seco, con precipitaciones que no sobrepasan los $500 \mathrm{~mm}$ anuales. Ambos distritos comparten la presencia de Aspidosperma quebracho-blanco Schltdl."quebracho blanco" y difieren en las especies de Schinopsis. Schinopsis balansae Engl. "quebracho colorado chaqueño" es característico del distrito oriental, Schinopsis lorentzii (Griseb.) Engl. "quebracho colorado santiagueño" caracteriza a la comunidad clímax del distrito occidental.

La recolección de plantas en flor se realizó durante la primavera y el verano de los años 20042007, totalizando 46 viajes de campaña. La toma de ejemplares se realizó en un radio de unos $2 \mathrm{~km}$ tomando al apiario como centro. Se colectaron principalmente aquellas plantas en las que se registró recolección de néctar por parte de las abejas, es decir las plantas que se esperaba encontrar en las muestras de miel. Los ejemplares fueron procesados según las técnicas convencionales de herborización (Rodríguez \& Rojas, 2006), luego fueron identificados taxonómicamente e incorporados al Herbario CTES del IBONE, UNNE-CONICET. A partir de esos ejemplares se realizaron preparaciones de polen de referencia que fueron incorporadas a la Palinoteca del IBONE (PAL-CTES).

Para detectar las plantas proveedoras de néctar se analizaron muestras de mieles tanto maduras como inmaduras. El análisis de las mieles inmaduras permite detectar las plantas utilizadas tempranamente por las abejas que por lo general no están representadas en las mieles maduras debido a que su néctar es consumido durante el período de expansión de la colonia. En cambio, las mieles maduras permiten identificar las plantas que contribuyen al mayor volumen de la cosecha. Las mieles inmaduras fueron extraídas directamente de los panales sin opercular por medio de una jeringa; en tanto que las mieles maduras fueron proporcionadas por los apicultores. Las mieles inmaduras fueron obtenidas en los años 2004-2005, en tanto que las mieles maduras entre los años 2004-2007.

Tanto el procesamiento como el análisis cualitativo de las mieles, se realizaron de acuerdo a la propuesta 


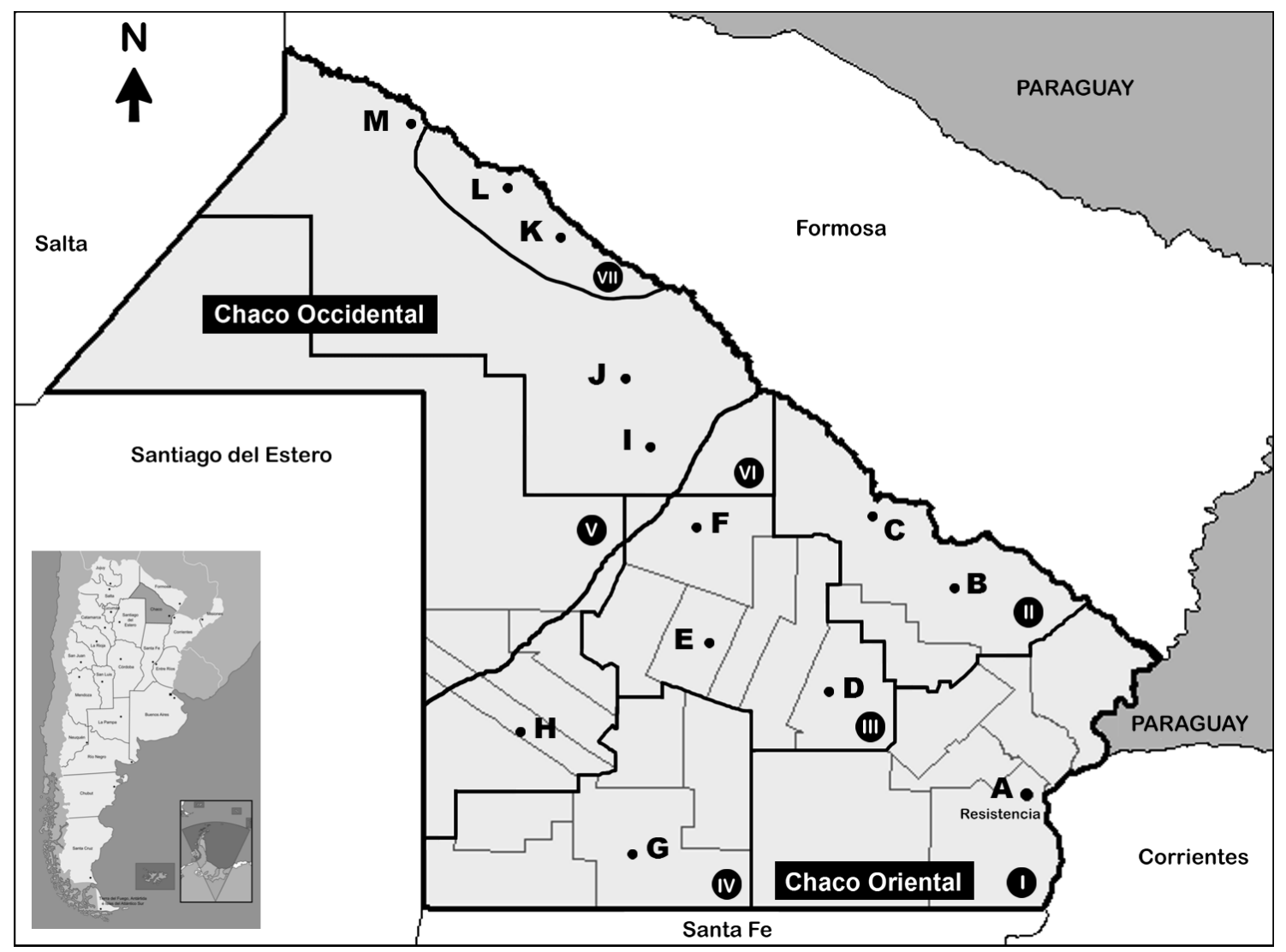

Fig. 1. Mapa de la ubicación de los apiarios muestreados. Chaco Oriental. A: Resistencia. B: Gral. San Martín. C: Gral. Roca. D: Presidencia Roque Sáenz Peña. E: Presidencia de la Plaza. F: Tres Isletas. G: Villa Ángela. H: Charata. Chaco Occidental. I: Juan José Castelli. J: Miraflores. K: Interfluvio, L. El Sauzalito y M. Tartagal.

de Louveaux et al. (1978), estableciendo las siguientes clases de frecuencia: polen dominante $\mathrm{D}(>45 \%$ del polen total), secundario $\mathrm{S}$ (entre 16 y $45 \%$ ); de menor importancia $\mathrm{M}$ (entre 3 y $15 \%$ ) y en traza $\mathrm{T}(<3 \%)$. El residuo polínico obtenido fue montado en preparados permanentes, posteriormente los granos fueron identificados, contados, estableciendo finalmente los porcentajes de los diferentes tipos morfológicos.

La importancia de las plantas como proveedoras de néctar fue establecida de acuerdo a su representatividad en las mieles.

\section{Resultados}

Registro de floración durante el periodo apícola

Se identificó un total de 282 especies colectadas a campo que se distribuyeron en 65 familias de
Angiospermas. Aquellas con mayor número de representantes (52\% del total) fueron: Asteraceae (52 especies), Fabaceae (37 especies), Solanaceae (19 especies), Verbenaceae (14 especies), Apiaceae (9 especies), Lamiaceae (8 especies) y Euphorbiaceae ( 8 especies), el $48 \%$ restante está representado por 58 familias con 1 a 6 representantes (Fig. 2).

El $91,5 \%$ de los taxones relevados es nativo y el 8,5\% restante son exóticos, incluyendo especies cultivadas y adventicias (Tabla 1).

En la curva de floración (Fig. 3) se destacan dos períodos de oferta que difieren en intensidad. Durante el primer periodo se obtiene la miel de primavera, que puede alcanzar un volumen de unas 560 toneladas y en el segundo periodo se obtiene la miel de verano que comúnmente ronda las 90 toneladas (Fuente: Ministerio de la Producción del Chaco). 

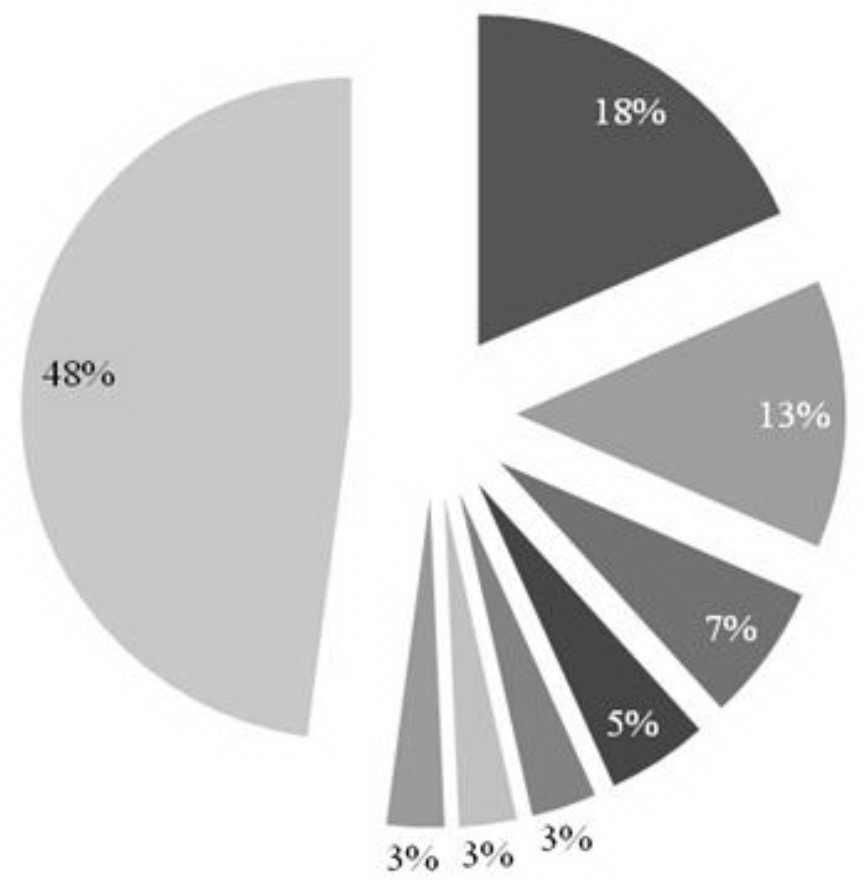

Asteraceae

Fabaceae

Solanaceae

Verbenaceae

Lamiaceae

Apiaceae

Euphorbiaceae

Otras

Fig. 2. Familias botánicas con mayor cantidad de representantes.

La floración se inicia en julio con muy pocas especies luego, entre agosto y septiembre la curva crece abruptamente alcanzando su máximo en octubre. A partir de noviembre la curva decrece, con una abrupta inflexión en enero; en febrero se incrementa nuevamente alcanzando un segundo pico de floración que comienza a declinar a partir de marzo. Se observó correspondencia entre los dos períodos de oferta y el hábito de las plantas que componen dicha oferta. Entre los meses de julio y enero, el mayor aporte lo realizan las plantas leñosas, en cambio a partir de enero y hasta mayo la mayor contribución la realizan las plantas herbáceas (Fig. 3).

\section{Recolección de néctar}

Se identificó un total de 118 tipos morfológicos, que representan el $42 \%$ de las especies relevadas.

El nivel de identificación de los tipos polínicos fue variable, 88 fueron identificados a especie, 21 a género y 2 a familia. Algunos taxones como Baccharis y Eupatorium no pudieron ser diferenciados debido a la similitud de sus caracteres polínicos, en ese caso se citan ambos.
El aporte de néctar en la región proviene de diversas familias botánicas aunque sólo unas pocas proveen el volumen necesario para la producción de mieles monoflorales, entre ellas: Anacardiaceae, Asteraceae, Fabaceae, Rhamnaceae y Zygophyllaceae. Representantes de otras familias que se encuentran como polen secundario, contribuyen a la producción de mieles mixtas, ellas son: Alismataceae, Apiaceae, Arecaceae, Brassicaceae, Capparaceae, Calyceraceae, Celastraceae, Celtidaceae, Cyperaceae, Euphorbiaceae, Lythraceae, Menyanthaceae, Myrtaceae, Phytolaccaceae, Polygonaceae, Pontederiaceae, Rutaceae, Sapotaceae, Solanaceae y Verbenaceae. Un elevado número de taxones que se encuentran escasamente representados en las mieles (i.e. como polen menor importancia o en traza) pertenecen a diversas familias como: Boraginaceae, Chenopodiaceae, Cyperaceae, Commelinaceae, Lamiaceae, Lauraceae, Limnocharitaceae, Malvaceae, Nyctaginaceae, Nymphaeaceae, Onagraceae, Oxalidaceae, Plantaginaceae, Ranunculaceae, Rubiaceae y Sapindaceae (Tabla 2). 


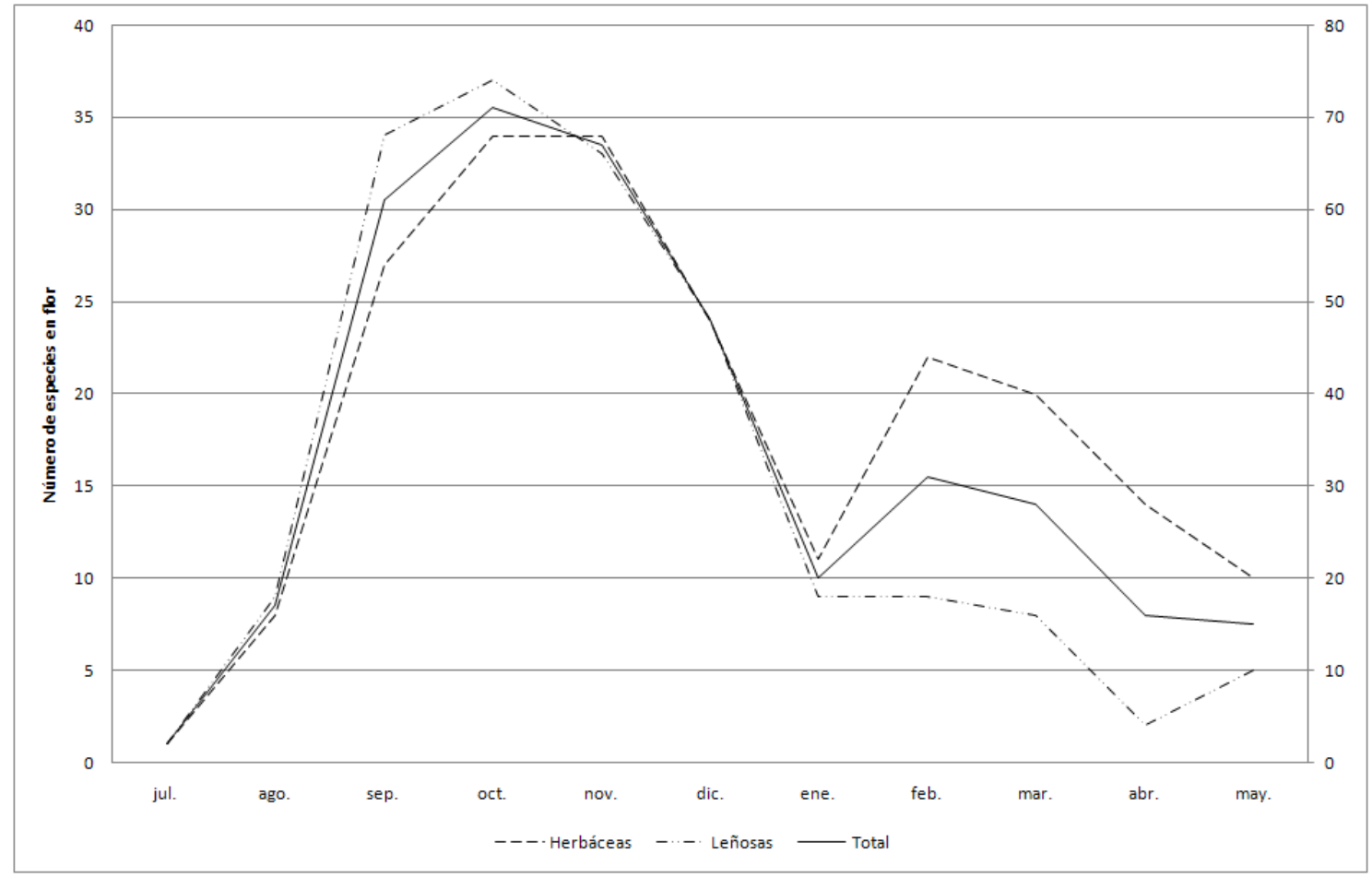

Fig. 3. Marcha anual de la floración.

La oferta de néctar se inicia en el mes de julio, la representatividad del polen en las mieles revela que las floraciones de Acacia praecox Griseb. "garabato negro", Geoffroea decorticans (Gillies ex Hook. \& Arn.) Burkart "chañar" y Prosopis alba Griseb. "algarrobo blanco" son muy utilizadas por las abejas. En particular, la floración de Prosopis alba provee néctar hasta principios de diciembre. Durante los meses de agosto y septiembre se suma el aporte de néctar de diversas herbáceas como Sagittaria montevidensis Cham. \& Schltdl. "saeta", Senecio grisebachii Baker "primavera", Acicarpha tribuloides Juss. "cardo torito", y Vicia macrograminea Burkart "arvejillas", acompañadas por una variedad de arbustivas y arbóreas Maytenus spinosa (Griseb.) Lourteig \& O'Donell y M. vitis - idaea Griseb. "sal del indio", Parkinsonia aculeata L. "cinacina", Eugenia uniflora L. "ñangapirí", Scutia buxifolia Reissek "coronillo", Ziziphus mistol Griseb. "mistol" y Salix humboldtiana Willd. "sauce". Las floraciones de Acicarpha tribuloides "cardo torito" y Sagittaria montevidensis "saeta", tienen una extensa duración, el polen de ambas especies se encuentra tanto en las mieles de primavera como en las de verano.

En octubre, se destacan las floraciones de las Arecaceae Copernicia alba Morong "palma" y Trithrinax schizophylla Drude "carandá" junto a Eryngium sp. y Helianthus annuus L. "girasol", cuyas floraciones llegan hasta el mes de noviembre. En diciembre se inicia la floración de diversas especies que continúan proveyendo néctar hasta el mes de marzo: Schinopsis balansae Engl. y $S$. lorentzii (Griseb.) Engl. "quebrachos colorados", Baccharis L. y Eupatorium L. "chilcas". Bulnesia sarmientoi Lorentz ex Griseb. "palo santo" y Tessaria integrifolia Ruiz \& Pav. "aliso de río".

La presencia en las mieles de polen proveniente de Amaranthus L. y Pfaffia Mart. (Amaranthaceae), Commelina erecta L. (Commelinaceae), Nymphaea L. (Nymphaeaceae), Paspalum notatum Flüggé (Poaceae) y Plantago sp. (Plantaginaceae) se debe a una contaminación, pues son plantas que no producen néctar. En un estudio de mieles de Patagonia, Forcone (2003b) atribuyó la presencia 


\begin{tabular}{|c|c|c|c|c|c|c|}
\hline \multirow{2}{*}{ Taxa } & \multicolumn{2}{|c|}{ Nativos } & \multicolumn{2}{|c|}{ Exóticos } & \multicolumn{2}{|c|}{ Totales } \\
\hline & Número & $\%$ & Número & $\%$ & Número & $\%$ \\
\hline Leñosos & 139 & 49,3 & 7 & 2,5 & 146 & 51,8 \\
\hline Herbáceos & 119 & 42,2 & 17 & 6,0 & 136 & 48,2 \\
\hline Total & 258 & 91,5 & 24 & 8,5 & 282 & 100 \\
\hline
\end{tabular}

de polen de plantas no nectaríferas en las mieles, a la acción del viento que llevaría el polen de estas plantas en suspensión, contaminando el néctar de otras flores que son utilizadas por las abejas.

\section{Discusión}

El estudio realizado puso en evidencia que en la región Chaqueña la floración se extiende durante la mayor parte del año, coincidiendo con Cabrera et al. (2013). Las floraciones provienen mayormente de especies nativas. Tanto la extensión del período, como el status de las plantas utilizadas (nativo o exótico), son caracteres distintivos de esta región. Otras provincias fitogeográficas argentinas donde las abejas también utilizan recursos nativos son la provincia del Monte (Naab \& Tamame, 2007), del Espinal (Gurini \& Basilio, 1995), en el distrito del Caldén (Andrada, 2003). En cambio, en otras regiones como la región Patagónica (Forcone, 2003a; Forcone \& Kutschker, 2006; Forcone \& Muñoz, 2009) y la Pampeana (Tellería, 1993) el período de floración es comparativamente más breve y además se destaca una intensa utilización de plantas introducidas. Si bien la oferta de plantas leñosas y herbáceas en la región estudiada fue similar durante todo el período muestreado, el polen proveniente de plantas leñosas domina en las muestras de miel. En general, estas plantas ofrecen mayor cantidad de recompensas debido a que poseen inflorescencias densas (e.g. Prosopis alba).

Dentro de las familias botánicas más importantes en la producción apícola del Chaco, se destacan Asteraceae y Fabaceae; esto también sucede en otras zonas productoras del país. En la primera se destacan: Baccharis, Eupatorium, Senecio, Vernonia y Tessaria. Con respecto a Fabaceae sobresalen las Mimosoideae como Acacia (Acacia aroma Gillies ex Hook. \& Arn., A. caven (Molina)
Molina y Prosopis alba, a diferencia de otras regiones argentinas donde las Faboideae son más relevantes (Forcone, 2003ab; Tellería 1993, 1995, 2000). Un lugar destacado como recurso de néctar en la producción chaqueña también lo ocupan las especies de Anacardiaceae típicas de la región como Schinopsis balansae y $S$. lorentzii, junto a Celastraceae representadas por especies de Maytenus Molina y Ziziphus mistol (Rhamnaceae), Myrtaceae (Eucalyptus sp.), Salicaceae (Salix humboldtiana) y Sapindaceae (Allophylus edulis (A. St.-Hil., A. Juss. \& Cambess.) Hieron. ex Niederl.).

Se registraron algunas diferencias en la oferta de floración en cada distrito; en el occidental se inicia tempranamente con la floración de Acacia praecox "garabato negro" y continúan en octubre y diciembre con las de Bulnesia sarmientoi "palo santo", Ziziphus mistol "mistol", Scutia buxifolia "coronillo" y Schinus fasciculatus (Griseb.) I.M. Johnst. "molle", continuando en enero y febrero con Schinopsis lorentzii (Griseb.) Engl. "quebracho colorado santiagueño", Tessaria integrifolia "aliso de rio" y Trithrinax schizophylla "carandá". En el distrito oriental el aporte de néctar comienza más tarde, las floraciones de Geoffroea decorticans "chañar", Parkinsonia aculeata "cina-cina", Vicia macrograminea "arvejillas", Senecio grisebachii "primavera" y Eryngium sp. "cardo". Cabe destacar que en este distrito existe una importante floración de cultivos que también son aprovechados por las abejas como Helianthus annuus "girasol" y Melilotus albus Desr. "meliloto". Esta última, junto a Eucalyptus sp., fueron consideradas representativas de las mieles chaqueñas y formoseñas (Basilio y Noetinger, 2002). Si bien la familia Myrtaceae es estenopalínica (i.e. homogénea por sus caracteres polínicos), en el presente trabajo, el seguimiento de la floración permitió concluir que no sólo Eucalyptus sp. es utilizado, sino también Eugenia uniflora "ñangapirí". Eucalyptus florece de diciembre a febrero, en cambio E. uniflora de agosto a noviembre (Tabla 2).

El contenido polínico de las mieles reflejó diferencias significativas en la utilización de los recursos nectaríferos. En el Distrito Occidental se destacaron las siguientes especies: Acacia praecox "garabato negro", Bulnesia sarmientoi "palo santo", Schinopsis lorentzii "quebracho 
Tabla 2. Período de floración de los taxones utilizados por las abejas melíferas para obtener néctar y polen. Período de floración y frecuencia de aparición en las mieles chaqueñas. D: polen dominante (> 45 $\%)$, S: polen secundario (16 - 45\%) M: polen de menor importancia (15 - 3\%), y T: polen en traza $(<3 \%)$. Las especies que presentan polen dominante y secundario se indican con color gris. La línea negra indica el período de mayor floración de la especie y el sombreado en gris claro indica el inicio y final de la floración. Las especies exóticas se indican con asterisco. Se indica con $\diamond$ las especies distintivas del Distrito Occidental.

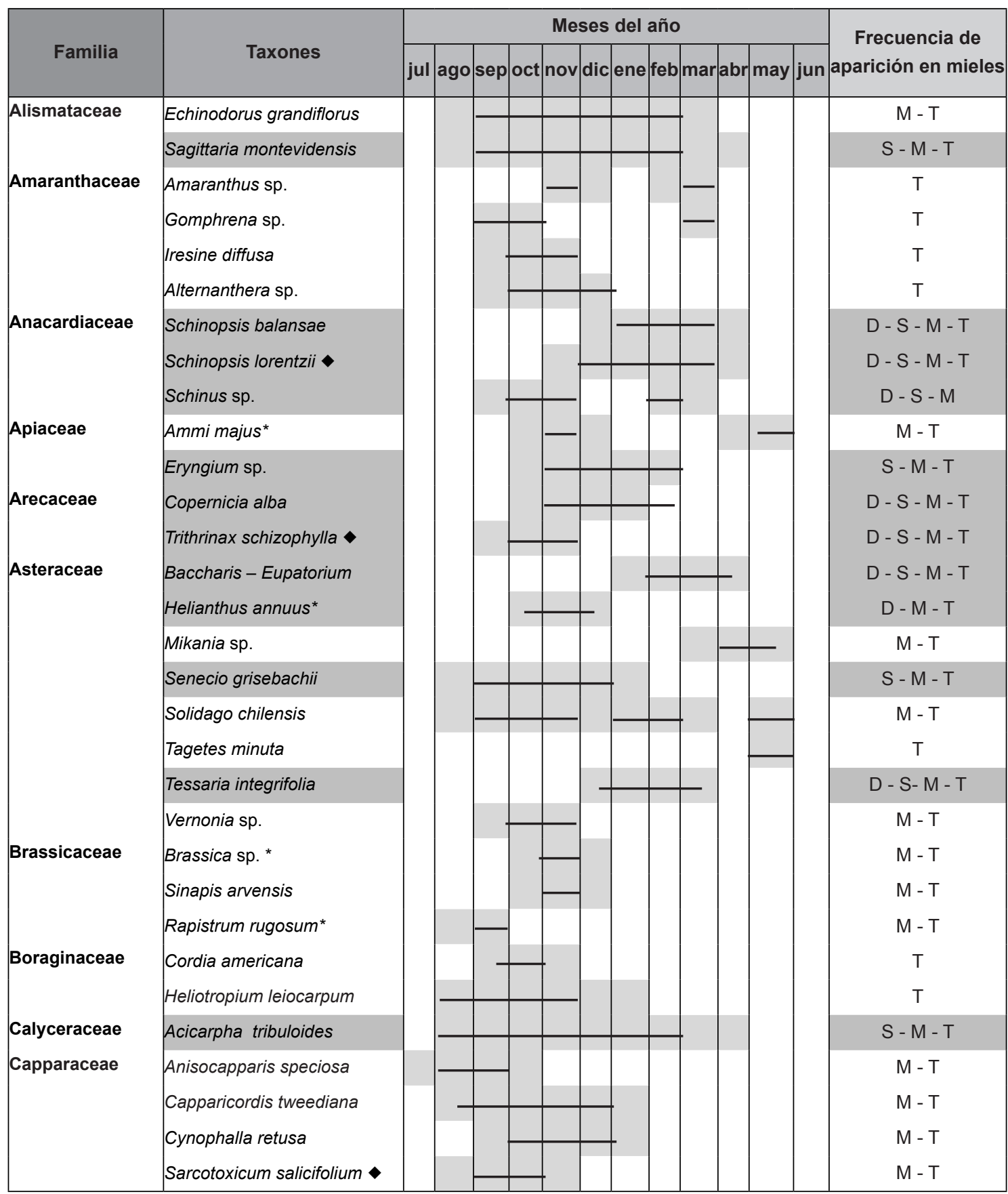


Bol. Soc. Argent. Bot. 49 (4) 2014

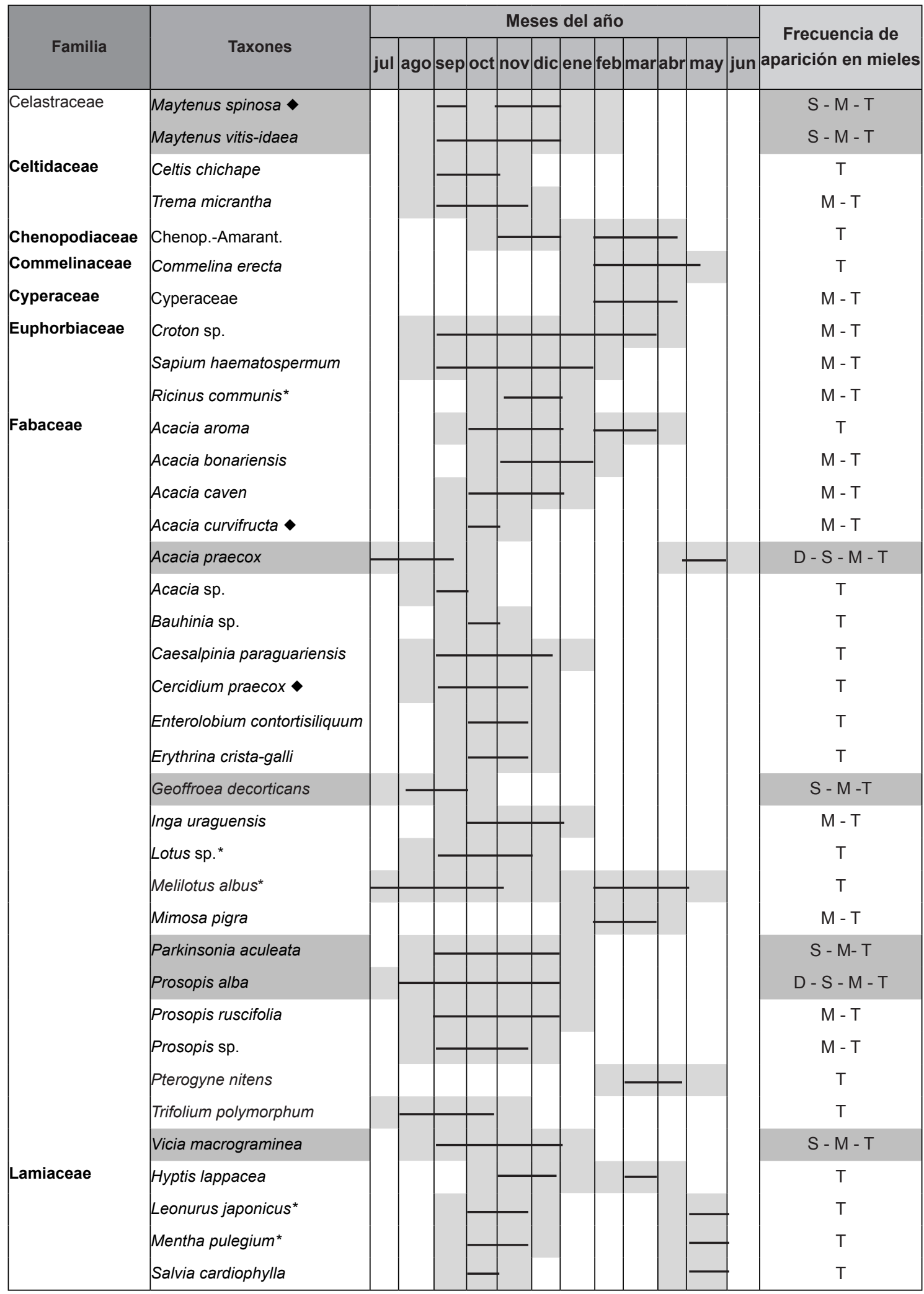


C. R. Salgado et al. - Aporte de la Melisopalinología al conocimiento de la flora Chaqueña

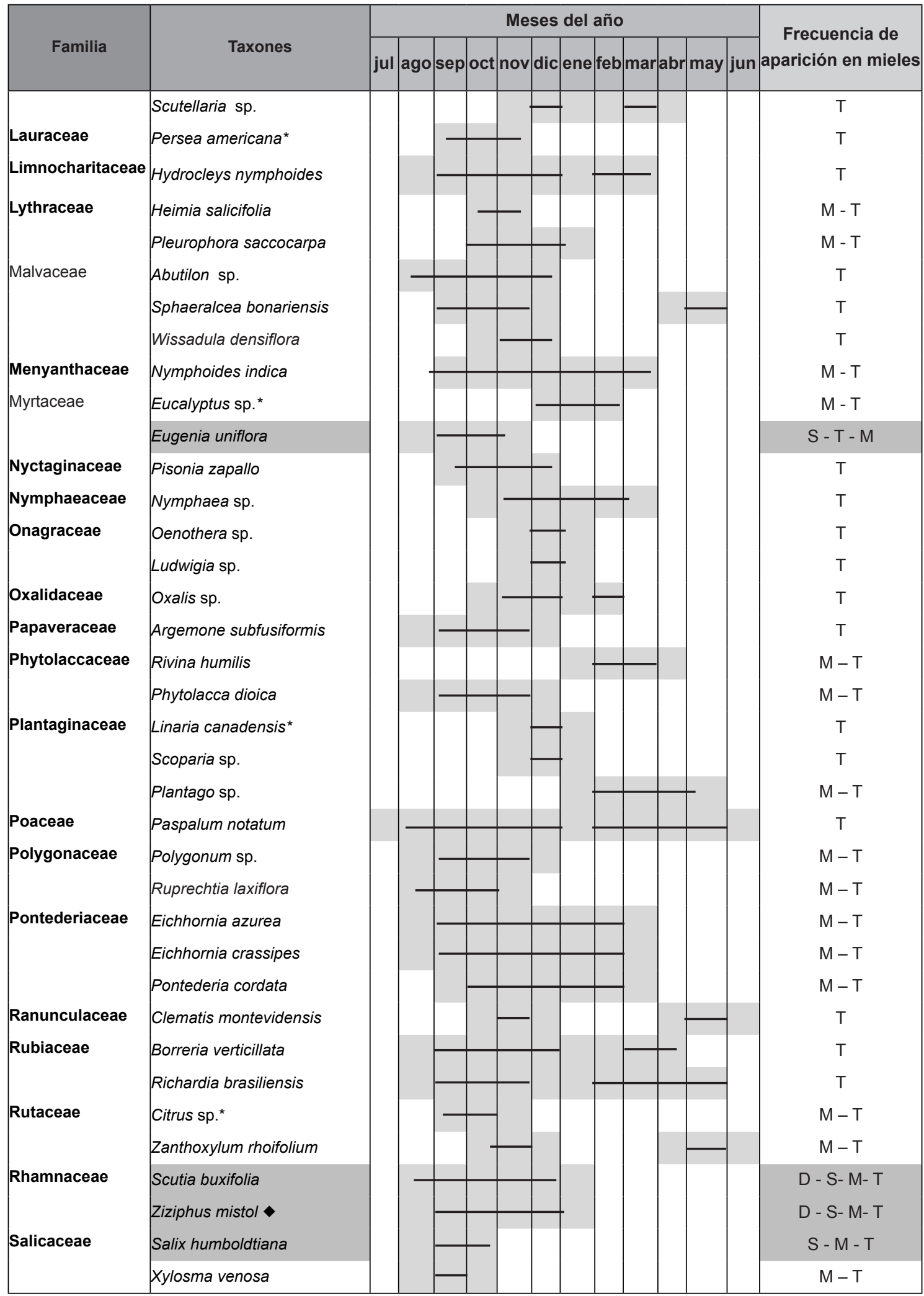


Bol. Soc. Argent. Bot. 49 (4) 2014

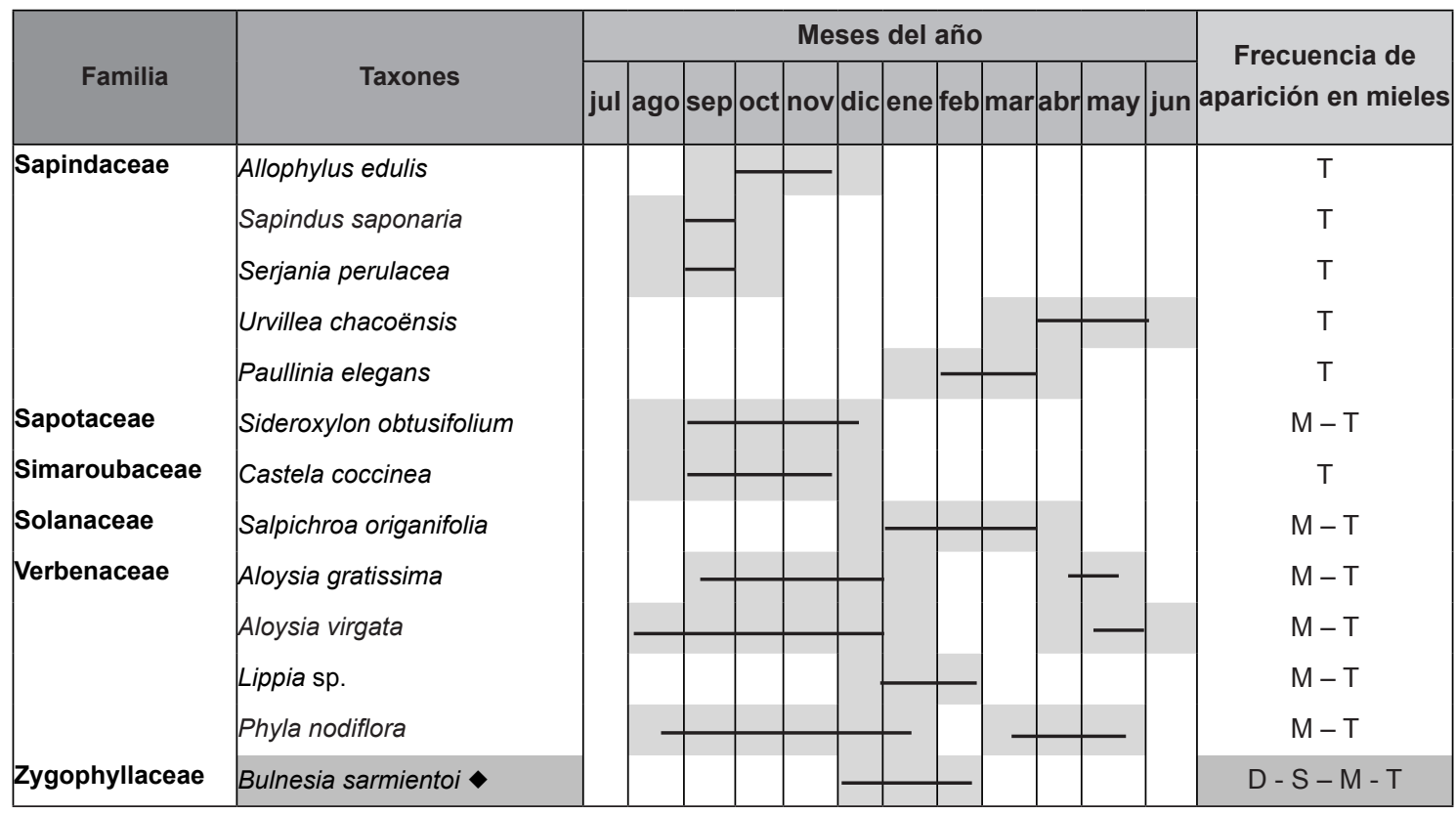

colorado santiagueño", Tessaria integrifolia "aliso de rio", Trithrinax schizophylla "carandá", Maytenus spinosa, M. vitis-idaea "sal del indio" y Ziziphus mistol "mistol". En el Distrito Oriental sobresalieron: Geoffroea decorticans "chañar", Eugenia uniflora L. "ñangapirí”, Helianthus annuus "girasol", Sagittaria montevidensis "saeta", Schinopsis balansae, y Copernicia alba "palma". Finalmente, algunos tipos polínicos mostraron una utilización similar en ambos Distritos de los siguientes taxones: Acicarpha tribuloides "cardo torito", Baccharis - Eupatorium, Prosopis alba, Parkinsonia aculeata "cina-cina", Senecio grisebachii "primavera" y Vicia macrograminea "arvejillas".

\section{Conclusiones}

El trabajo realizado reafirmó observaciones previas, a la vez que aportó información inédita sobre la flora chaqueña de interés apícola. Como fue observado por Cabrera et al. (2013) el potencial nectarífero de la región reside en la flora nativa, principalmente en las especies leñosas, además el período de oferta de floración es extenso comparado con otras regiones argentinas. Sin embargo, el estudio de las mieles acompañado por el seguimiento de la floración permitió sumar la siguiente información:

- el período de disponibilidad de néctar se inicia tempranamente, en el mes de agosto, en el Distrito Occidental y extendiéndose hasta el mes de marzo en el Distrito Oriental.

- existen dos "momentos" de mayor oferta de floración, una de mayor envergadura en la primavera y otra menor en el verano.

- las especies a partir de las cuales se pueden producir mieles monoflorales en primavera son: Acacia praecox y Prosopis alba (Fabaceae) y en las de verano: Schinopsis balansae y S. lorentzii (Anacardiaceae), Copernicia alba (Arecaceae), Baccharis y Eupatorium, Tessaria integrifolia (Asteraceae), Bulnesia sarmientoi (Zygophyllaceae) y Ziziphus mistol (Rhamnaceae).

En síntesis, el trabajo realizado contribuye a profundizar el conocimiento de los recursos nectaríferos de la región chaqueña destacando condiciones muy favorables para la actividad como un período de floración extenso y una importante oferta de recompensas provenientes de plantas nativas. Esto último posibilita el aprovechamiento de floraciones de plantas que no están sujetas a tratamientos químicos (pesticidas, etc.), condición 


\section{R. Salgado et al. - Aporte de la Melisopalinología al conocimiento de la flora Chaqueña}

valorada en el marco de la producción de mieles orgánicas bajo un protocolo de certificación. En este sentido es importante destacar que la vegetación de la región estudiada tiene aptitud para la producción apícola de particular interés para los países importadores de mieles argentinas.

\section{Agradecimientos}

Las autoras desean expresar su agradecimiento por el apoyo económico para la realización del trabajo a través de un PI otorgado por la SGCyT de la UNNE. A la Dra. María Mercedes Arbo, Curadora del herbario CTES, por permitir el ingreso de las plantas y su posterior incorporación a la colección. Al Ministerio de la Producción de la provincia del Chaco por colaborar en la logística de los viajes de colección y muestreo. A Sandra Sobrado por la edición del mapa.

\section{Bibliografía}

ANDRADA, A. 2003. Flora utilizada por Apis mellifera L. en el sur del Caldenal (Provincia Fitogegráfica del Espinal), Argentina. Rev. Mus. Argentino Cienc. Nat., ns. 5: 329-336.

BASILIO, A.M. \& M. NOETINGER. 2002. Análisis polínico de mieles de la región Chaqueña: comparación del origen floral entre las zonas, domo central y esteros, cañadas y selvas de ribera. RIA (Revista de Investigaciones Agropecuarias) 31: 127-134.

CABRERA, A. L. 1976. Regiones fitogeográficas de la República Argentina, en Enciclopedia Argentina de Agricultura y Jardinería 2(1). ACME, Buenos Aires.

CABRERA, M.M. 2006. Caracterización polínica de las mieles de la Provincia de Formosa, Argentina. Rev. Mus. Argen. Cienc. Nat. 8: 135-142.

CABRERA, M.M.; ANDRADA, A. \& L. GALLEZ. 2013. Floración de especies con potencial apícola en el Bosque Nativo Formoseño, Distrito Chaqueño Oriental (Argentina). Bol. Soc. Argent. Bot. 48: 477-491.

DINERSTEIN, E.D.; OLSON, M.; GRAHAM, D.J. WEBSTER, A.L.; PRIMM, S.A.; BOOKBINDER, M.P. \& G. LEDEC. 1995. Una evaluación del estado de conservación de las ecorregiones terrestres de América Latina y el Caribe. Wold Bank, Washington, D.C.
FORCONE, A. 2003a. Floración y Utilización de la Flora Apícola en el Valle Inferior del Río Chubut (Patagonia-Argentina). Bol. Soc. Argent. Bot. 38: 301-317.

FORCONE, A. 2003b. Plantas nectaríferas utilizadas por Apis mellifera L. en la Patagonia extra andina, Argentina. Rev. Mus. Argen. Cienc. Nat. 5: 363-369

FORCONE, A. \& A. ANDRADA, 2007. Flora melífera de las regiones pampeana austral y Patagonia extraandina. Editorial de la Universidad Nacional del Sur - EDIUNS, Red de Editoriales Universitarias Nacionales - REUN. 173 págs 1ra Ed. ISBN-10: 987-1171-60-9

FORCONE, A. \& A. KUTSCHKER, 2006. Floración de las especies de interés apícola en el noroeste de Chubut, Argentina. Rev. Mus. Argen. Cienc. Nat. 8: 151-157.

FORCONE, A. \& M. MUÑOZ, 2009. Floración de las especies de interés apícola en el noroeste de Santa Cruz, Argentina. Bol. Soc. Argent. Bot. 44: 393-403.

GURINI, L. \& A.M. BASILIO, 1995. Flora apícola en el Delta del Paraná. Darwiniana 33: 337-346.

LOUVEAUX, J., MAURIZIO, A. \& G.VORWOHL, 1978. Methods of Melissopalynology. Bee World 59: 139-157.

MAIDANA, J. 1976. Determinación de la flora melífera del departamento Capital de la Provincia de Corrientes. Trabajo de graduación. Facultad de Ciencias Agrarias. Universidad Nacional del Nordeste. Inédito.

MENDEZ, E. 1997. Flora melífera de la carta de vegetación Zapata (Mendoza- Argentina). Bol. Soc. Argent. Bot. 33:7-12.

NAAB, O \& M. A. TAMAME. 2007. Flora apícola primaveral en la región del Monte de la provincia de la Pampa (Argentina). Bol. Soc. Argent. Bot. 42: 251-259.

RODRÍGUEZ, E.F. \& R.P. ROJAS. 2006. El herbario. Administración y manejo de colecciones botánicas. Herbarium Truxillense (Hut) Missouri Botanical Garden. Facultad de Ciencias Biológicas. Universidad Nacional de Trujillo. Ed. Rodolfo Vásquez.

SALGADO, C.R. 2006. Flora melífera de la provincia de Chaco. Editado por PROSAP y Ministerio de Producción del Chaco. pp: 60.

SANCHEZ, A.C. \& C.L. LUPO. 2011. Origen botánico y geográfico de las mieles de El Fuerte, Departamento de Santa Bárbara, Jujuy, Argentina. Bol. Soc. Argent. Bot. 46: 105-111.

SÁNCHEZ, A.C. \& N.D. VIGNALE. 2009. Flora apícola de la Quebrada de Humahuaca. Arnaldoa 16: 101-108. 
Bol. Soc. Argent. Bot. 49 (4) 2014

TELLERIA, M.C. 1993. Floraison et récolte do pollen dans la pampa argentine. Apidologie 24: 109-121.

TELLERIA, M.C. 1995. Plantas de importancia apícola del Distrito Oriental de la Región Pampeana (Argentina). Bol. Soc. Argent. Bot. 30: 131-136.

TELLERIA, M.C. 2000. Contribución a la identificación del polen de las mieles pampeanas. Bol. Soc. Argent. Bot. 34: 205-216.

Recibido el 24 de marzo de 2014, aceptado el 10 de julio de 2014. 